\section{ORIGINAL RESEARCH}

\author{
D. Kaya \\ A. Dinçer \\ M.E. Yıldız \\ M.O. Çizmeli \\ C. Erzen
}

\title{
Acute Ischemic Infarction Defined by a Region of Multiple Hypointense Vessels on Gradient-Echo T2* MR Imaging at 3T
}

\begin{abstract}
BACKGROUND AND PURPOSE: During the hyperacute phase of stroke, multiple hypointense vessels were identified specifically in the ischemic territory on gradient-echo T2*-weighted MR images (GRE-T2* WI) at 3T. The area was named a "region of multiple hypointense vessels (RMHV)." The aim of this study was to assess the usefulness of RMHV for the diagnosis of acute ischemic stroke (AIS) and to establish the relationship of this finding to other MR imaging studies.
\end{abstract}

\begin{abstract}
MATERIALS AND METHODS: Twenty patients with AIS underwent MR imaging at $3 T$ consisting of GRE-T2*, diffusion-weighted images (DWI), and perfusion-weighted images (PWI) within 6 hours of symptom onset and follow-up images at 72 hours. RMHV was defined as an area containing multiple hypointense vessels strictly in the region of the ischemic territory on GRE-T2*. The RMHV volume on GRE-T2* initial ischemic lesion volumes on DWI, PWI maps, and on follow-up images were measured and compared with the RMHV volume.
\end{abstract}

RESULTS: RMHV on GRE-T2* was identified in 20 patients. There was no significant difference between the ischemic lesion volumes on mean transit time $(247.3 \pm 88.1 \mathrm{~mL})$, time-to-peak $(228.6 \pm$ $88.8 \mathrm{~mL})$, cerebral blood flow $(200.6 \pm 89.7 \mathrm{~mL}), \mathrm{RMHV}$ on GRE-T2* $(214.4 \pm 86 \mathrm{~mL})$, and the infarct volume at 72 hours $(210.3 \pm 90.4 \mathrm{~mL})(P=.975)$.

CONCLUSIONS: RMHV on GRE-T2* can be used as a supportive imaging finding for the diagnosis of hyperacute ischemic stroke. RMHV volume provides information that is in accordance with the infarct volume at 72 hours and the data supplied by PWI.

$\mathbf{R}$ ecent studies have demonstrated that multiparametric MR imaging protocols offer an additional source of data regarding tissue status and identify patients with stroke who may benefit from intravenous thrombolysis. Gradient-echo $\mathrm{T} 2^{\star}$-weighted image (GRE-T2* WI) is a sequence sensitive to paramagnetic substances such as deoxyhemoglobin, which becomes a naturally occurring endogenous contrast agent for MR imaging. ${ }^{1,2}$ This MR image can be applied to exclude hemorrhage $^{3}$ but can also show intra-arterial clot, which is seen as hypointense signal intensity in an artery and is called the GRE susceptibility vessel sign (GRE-SVS) ${ }^{4}$ in acute ischemic stroke (AIS). Furthermore "hypointense venous signal intensity loss area" has been described by use of 1.5 T GRE MR imaging in some cases of AIS in animals ${ }^{5}$ and human studies ${ }^{6-8}$ related to the abnormal visibility of transcerebral veins or abnormal visualization of leptomeningeal vessels. We observed a similar finding of multiple venous or arterial hypointense vessels strictly confined to the ischemic territory in all patients who had large cerebral arterial occlusion in the hyperacute phase of the ischemic stroke at 3T GRE MR imaging.

The area containing the linear and branching signal intensity-void structures on GRE-T2* was named the region of multiple hypointense vessels (RMHV). The main aim of this study was to assess the usefulness of RMHV for the diagnosis

Received December 15, 2008; accepted after revision January 8, 2009.

From the Departments of Neurology (D.K.) and Radiology (A.D., M.E.Y., M.O.C.., C.E.), Acibadem University School of Medicine, Istanbul, Turkey.

Please address correspondence to Dilaver Kaya, MD, Acibadem Kozyatagi Hastanesi, Noroloji Bolumu, Inonu cad., Okur sok. 20, Kozyatagi 34742, Istanbul, Turkey; e-mail: dilaverka@yahoo.com

Indicates open access to non-subscribers at www.ajnr.org

DOI 10.3174/ajnr.A1537 of acute ischemic stroke and to determine the relationship of this finding to other established MR studies in AIS.

\section{Materials and Methods}

\section{Patients}

From November 2006 to August 2008, a total of 67 consecutive patients with acute stroke were evaluated with $3 \mathrm{~T}$ MR imaging protocol within 6 hours after symptom onset. The following data were recorded: patient age, sex, location of infarction, and time of onset of clinical symptoms at admission to the hospital. Stroke severity was assessed according to the National Institutes of Health Stroke Scale (NIHSS) score. The following criteria were established for inclusion in the study: 1) baseline NIHSS score of more than 4, and 2) largeartery occlusion on time-of-flight MR angiography (TOF-MRA). Intravenous (IV) thrombolysis with recombinant tissue plasminogen activator (rtPA) was given within 3 hours after symptom onset at the usual dose of $0.9 \mathrm{mg} / \mathrm{kg} .{ }^{9}$ Eligibility criteria for rtPA administration at our hospital included the conventional National Institute of Neurologic Disorders and Stroke criteria ${ }^{9}$ (single exception: patients older than 80 years were not treated by rtPA), with the addition of MR imaging criteria (perfusion/diffusion mismatch). Informed consent was obtained from patients or their relatives. This prospective study protocol was approved by the hospital ethics committee. There were 30 of 67 patients who had acute large cerebral artery occlusion, 7 of 30 patients had transient ischemic attack, and 3 of 30 patients had movement artifact on the MR imaging sequences. Therefore, only 20 patients were finally included in the study. There were 12 of 20 patients who were admitted to the hospital within 3 hours after symptom onset and 8 of 20 patients within 3 to 6 hours. Six of 12 patients were treated with IV rtPA, but the other 6 patients were not treated with rtPA because perfusion/diffusion match was observed in 2 patients, a co-incidental aneurysm was found in 1 patient, a large diffusionweighted image (DWI) lesion was observed in 1 patient, malignant 
hypertension $(240 / 130 \mathrm{~mm} / \mathrm{Hg})$ was found in 1 patient, and the sixth patient was 87 years old.

\section{Imaging Sequences}

3T MR imaging (Trio; Siemens, Erlangen, Germany) acute stroke protocol parameters were as follows: 3D TOF-MRA: TR, $19 \mathrm{~ms}$; TE, $3.69 \mathrm{~ms}$; flip angle (FA), $18^{\circ}$; matrix, $177 \times 256$; FOV , $165 \times 220 \mathrm{~mm}$; partition/slab thickness, $64 / 72 \mathrm{~mm}$; NEX, 1 ; acquisition time (AT), $71 \mathrm{~s}$. The parameters for the turbo spin-echo (TSE) T2WI were TR, $3500 \mathrm{~ms}$; TE, $85 \mathrm{~ms}$; matrix, $153 \times 256$; FOV, $178 \times 220 \mathrm{~mm}$; NEX, 1 ; AT, $40 \mathrm{~s}$. The parameters for the TSE T1WI were TR, $400 \mathrm{~ms}$; TE, 12 ms; matrix, $144 \times 256$; FOV, $177 \times 230 \mathrm{~mm}$; NEX, 1 ; and AT, $36 \mathrm{~s}$. The parameters for the fluid-attenuated inversion recovery (FLAIR) images were TR, $7620 \mathrm{~ms}$; TE, $82 \mathrm{~ms}$; matrix, $156 \times 256$; FOV, $177 \times$ $230 \mathrm{~mm}$; NEX, 1; and AT, $70 \mathrm{~s}$. The imaging parameters for the GRET2* fast low-angle shot (FLASH) images were TR, $400 \mathrm{~ms}$; TE, $12 \mathrm{~ms}$; matrix, $180 \times 320$; FOV, $177 \times 230 \mathrm{~mm}$; NEX, 1; and AT, $74 \mathrm{~s}$. The parameters for the contrast-enhanced cervical MR angiography (MRA) 3D FLASH T1 images were TR, $2.91 \mathrm{~ms}$; TE, $1.2 \mathrm{~ms}$; matrix, $256 \times 300$; FOV, $225 \times 300 \mathrm{~mm}$; FA, 15; voxel size, $0.8 \times 0.8 \times 0.8$ $\mathrm{mm}^{3}$; bandwidth, $930 \mathrm{~Hz} / \mathrm{px}$; sections per slab, 80; partition thickness, 0.8 . The imaging parameters for the DWI studies were TR, 3100 ms; TE, 106 ms; matrix, $128 \times 128$; FOV, $196 \times 196$ mm; NEX, 3; AT, $85 \mathrm{~s}$; and $2 \mathrm{~b}$-values $\left(1000\right.$ and $\left.2000 \mathrm{~s} / \mathrm{mm}^{2}\right)$. The latter applied in each of the 3 principal gradient directions $(\mathrm{x}, \mathrm{y}$, and $\mathrm{z})$ were used to calculate the apparent diffusion coefficient (ADC). We obtained perfusion-weighted images (PWI) using a bolus of gadobenate dimeglumine (MultiHance; Bracco Diagnostics, Princeton, NJ), $0.1 \mathrm{mmol} / \mathrm{kg}$ injected at $4 \mathrm{~mL} / \mathrm{s}$, and followed by $30 \mathrm{~mL}$ of saline. PWI was performed with spin-echo echo-planar imaging (EPI) sequence with the following parameters: TR, $1360 \mathrm{~ms}$; TE, $31 \mathrm{~ms}$; matrix, $128 \times 128$; FOV , $230 \times 230 \mathrm{~mm}$; NEX, 1; and AT, $78 \mathrm{~s}$. We obtained parametric perfusion maps including mean transit time (MTT), time-to-peak (TTP), and relative cerebral blood flow ( $\mathrm{rCBF}$ ) and volume (rCBV) using commercially available software supplied by the vendor. After a suitable basic image for positioning of the arterial input function (AIF) is selected, region of interest is drawn on healthy arteries close to the perfusion anomaly. The anterior cerebral artery (ACA) is usually selected for AIF. After selection of suitable AIF curves, the mean AIF was determined. The time range is set on the mean AIF curve. After the time range is confirmed, MTT, TTP, rCBF, and $\mathrm{rCBV}$ are calculated and displayed. All sequences were obtained with 5-mm section thickness and 1-mm intersection gap.

Baseline scanning protocol was standardized and included T2WI, T1WI, DWI, TOF-MRA, FLAIR, and GRE-T2*. Baseline scans were obtained in all patients. If the patient had a large artery occlusion on TOF-MRA, PWI was added for evaluation of thrombolysis within 3 hours. To identify infarct volume at 72 hours, follow-up imaging with a T2-weighted MR imaging or 16-section row CT scanner (Sensation 16; Siemens, Malvern, $\mathrm{Pa}$ ) was performed within 72 hours after symptom onset. All subjects had baseline and follow-up scans, but only 13 of 20 patients had initial PWI. Only 1 patient had an initial contrast-enhanced cervical MRA.

\section{Image Analysis}

Two independent experienced radiologists, A.D. (16 years' experience) and M.E.Y. (7 years' experience), blinded to each other's outlines and to clinical information concerning the patients visually identified and sequentially outlined regions abnormal on DWI $(b=$ 2000), PWI, and GRE-T2* in baseline MR imaging and follow-up images (T2-weighted MR imaging in 14 patients and CT in 6 patients). Areas of increased signal intensity on DWI and decreased signal intensity on ADC maps were classified as acute infarct territory. Regions with hypoperfusion on PWI were identified as areas of increased signal intensity on MTT and TTP maps and as decreased signal intensity on $\mathrm{rCBF}$ and $\mathrm{rCBV}$ maps. RMHV was defined on the baseline GRE-T2* -weighted sequence as an area that contains multiple hypointense linear and branching vessels, extending through the cortex and subcortical regions of the ischemic territory (Figs 1 and 2). To identify the borders of the RMHV on GRE-T2* and measure the area containing these vascular structures, we marked the tips of the prominent vessels lying at the most medial, anterior, and posterior border, and we drew lines between these markings. Thus, segmentation was performed on each section where the prominent vessels were identified, and from the segmented areas, the total volume of the RMHV was calculated. Regions with increased signal intensity on follow-up T2-weighted images or decreased attenuation areas on CT scans were considered as the infarct region at 72 hours. All areas of ischemic territory were segmented on a section-by-section basis by manual editing. The volumes were automatically produced by the software (MRIcro; Chris Rorden, University of South Carolina, Columbia, SC) on the basis of the section thickness and overall outlined lesion areas. RMHV was absent on the normal contralateral hemisphere of patients with stroke. GRE-T2* images were also analyzed for the presence of SVS.

\section{Statistical Analysis}

We analyzed the data using Statistical Package for Social Sciences (SPSS, Chicago, Ill) software (version 11.5). Friedman ANOVA was used for descriptive values and volumetric comparisons. Intraclass correlation coefficients (ICC) were computed to evaluate the correlation between examiners in lesion volume measurements on DWI, GRE-T2*, PWI maps, and follow-up images. The Bland-Altman plot was used for absolute difference between examiners. ${ }^{10,11}$ The Spearman correlation coefficient was used to analyze the relationship between lesion volumes and NIHSS score. The infarct volume at 72 hours of patients who received thrombolysis was not included for statistical analysis. Patient 5 had a large hemispheric hypoperfusion on PWI maps. The data of this patient were also not included in the statistical analysis because of acute occlusion of the angular branch (M3) of the middle cerebral artery (MCA) with concomitant ipsilateral severe stenosis of the proximal internal carotid artery (ICA). Patients were divided into 3 groups for volumetric measurement comparisons. The groups were structured according to availability of initial PWI:

1) Group 1 (patients 7-13) with initial DWI, PWI, GRE-T2*, and follow-up images.

2) Group 2 (patients 7-20) with initial DWI, GRE-T2*, and follow-up images.

3) Group 3 (patients $1-4$ and patients 6-13) with initial DWI, PWI, and GRE-T2* images.

\section{Results}

The study population included 13 men and 7 women. The mean age of the study population was 71.8 years (range, 37-90 years), median initial NIHSS score was 13.14 (range, 6-27). Symptomatic vascular occlusions were categorized as occurring in the ICA in 6 patients, in the MCA in 11 patients (M1, 6; $\mathrm{M} 2,1 ; \mathrm{M} 3,4)$, in the ACA in 1 patient, in the posterior cerebral artery in 1 patient, and in the basilar artery in 1 patient as 

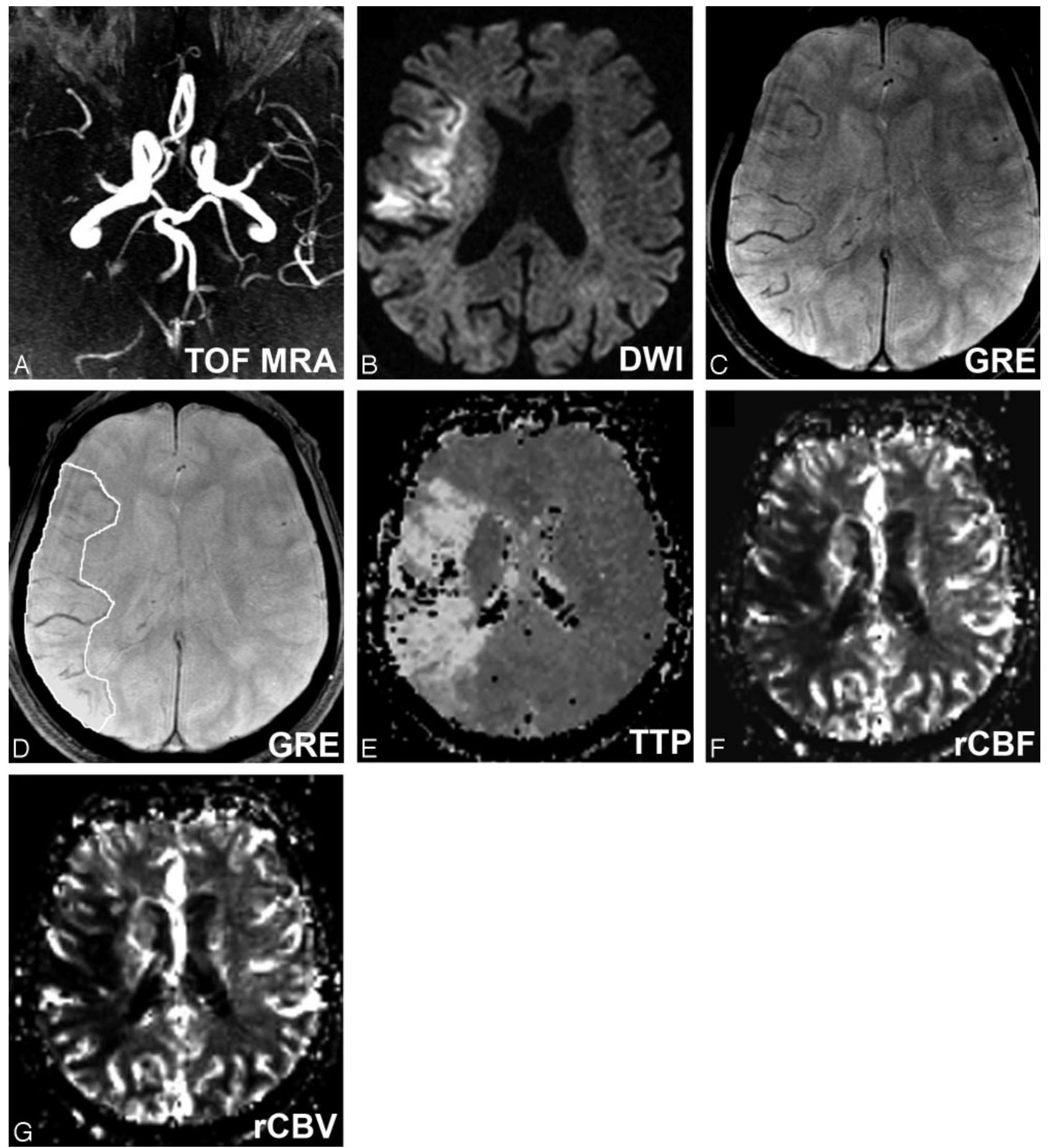

Fig 1. A, Craniocaudal collapsed MIP image of 3D TOF-MRA shows right MCA occlusion in case 2. B, Axial DWI-b2000 trace image shows hyperintensity in corresponding ischemic territory C, Axial GRE-T2* shows RMHV as linear or branching structures strictly in the ischemic territory. Please note that the contralateral hemisphere is free of such structures. $D$, Axial GRE-T2* shows outlined RMHV on the same image. TTP $(E)$, rCBF $(F)$, and rCBV $(G)$ maps show hypoperfusion area in corresponding ischemic territory.

diagnosed on 3D TOF-MRA. SVS and RMHV were identified on GRE-T2* in all patients (Table).

Group $1(\mathrm{n}=7)$

The mean $( \pm \mathrm{SD})$ baseline whole DWI lesion volume was $91.6 \pm 29.2 \mathrm{~mL}$ (range, 25.8-93.2 mL), MTT was 247.3 \pm 88.1 $\mathrm{mL}$ (range, 52.3-259.2 mL), TTP was $228.6 \pm 88.8 \mathrm{~mL}$ (range, 46.2-274.5 mL), rCBF was $200.6 \pm 89.7 \mathrm{~mL}$ (range, 35.3-276 $\mathrm{mL}$ ), rCBV was $175.3 \pm 71.9 \mathrm{~mL}$ (range, 28.6-222.3 mL),
RMHV on GRE-T2* was $214.4 \pm 86 \mathrm{~mL}$ (range, 46.8 -to 269 $\mathrm{mL}$ ), and the infarct volume at 72 hours was $210.3 \pm 90.4 \mathrm{~mL}$ (range, 49.5-284.7 mL). There was no significant difference between MTT, TTP, rCBF, and RMHV on GRE-T2* ${ }^{\star}$, and the infarct volume at 72 hours $(P=.975)$.

\section{Group $2(\mathrm{n}=14)$}

The mean $( \pm \mathrm{SD})$ baseline DWI lesion volume was $86.2 \pm 41.5$ $\mathrm{mL}$ (range, $16.3-176.2 \mathrm{~mL}$ ), RMHV on GRE-T2* was $217.7 \pm$ 



Fig 2. Right ICA occlusion in case 15. A, DWI-b2000 image shows small hyperintensities in the right posterior temporal and anterior parietal lobes, the caudate body and head, the right putamen and globus pallidus, and the internal and external capsules. $B$, Axial GRE-T2* corresponding image shows large hemispheric RMHV in the ischemic territory. $C$, Follow-up CT image shows the infarcted area at 72 hours.

\begin{tabular}{|c|c|c|c|c|c|c|c|c|c|c|c|c|c|c|c|c|}
\hline \multicolumn{17}{|c|}{ Patient data and volumetric measurements } \\
\hline \multicolumn{3}{|c|}{ Patients } & \multicolumn{2}{|c|}{ Admission } & \multicolumn{2}{|c|}{ Occlusion } & \multirow[b]{2}{*}{$\begin{array}{l}\text { GRE } \\
\text { (SVS) }\end{array}$} & \multirow[b]{2}{*}{ tPA } & \multicolumn{6}{|c|}{ Initial Lesion Volumes (mL) } & \multicolumn{2}{|c|}{ Follow-up } \\
\hline No & Age & $S$ & $\begin{array}{l}\text { Time } \\
(\min )\end{array}$ & NIHSS & Arteries & Location & & & DWI & TTP & MTT & $\mathrm{rCBF}$ & rCBV & $\begin{array}{l}\text { RMHV on } \\
\text { GRE }\end{array}$ & Images & $\begin{array}{l}\text { Volumes } \\
(\mathrm{mL})\end{array}$ \\
\hline 1 & 74 & $\mathrm{M}$ & 75 & 16 & ICA & Right & Yes & Yes & 61.6 & 300 & 314.5 & 210.6 & 162.8 & 255.1 & T2 & 153.7 \\
\hline 2 & 70 & M & 60 & 13 & MCA (M1) & Right & Yes & Yes & 55.5 & 230 & 245.7 & 215.6 & 171.6 & 241.1 & $\mathrm{~T} 2$ & 76.7 \\
\hline 3 & 74 & M & 60 & 12 & MCA (M1) & Right & Yes & Yes & 41.5 & 329.2 & 334.8 & 307.9 & 256.1 & 311.5 & T2 & 34.5 \\
\hline 4 & 77 & $\mathrm{~F}$ & 140 & 14 & MCA (M1) & Right & Yes & Yes & 106 & 203.7 & 207.3 & 173.1 & 153.8 & 199.9 & $\mathrm{~T} 2$ & 127.2 \\
\hline 5 & 71 & $\mathrm{~F}$ & 80 & 6 & MCA (M3) & Left & Yes & Yes & 6.1 & 281.4 & 233.6 & 176.9 & 185.4 & 70.7 & $\mathrm{~T} 2$ & 3.4 \\
\hline 6 & 76 & $\mathrm{~F}$ & 70 & 12 & MCA (M2) & Left & Yes & Yes & 34.4 & 54.8 & 61.6 & 62.7 & 37.5 & 60 & T2 & 32.1 \\
\hline 7 & 87 & $\mathrm{~F}$ & 150 & 14 & MCA (M1) & Right & Yes & No & 93.2 & 228.6 & 247.3 & 200.6 & 142 & 198 & T2 & 206.6 \\
\hline 8 & 75 & $\mathrm{~F}$ & 50 & 21 & ICA & Left & Yes & No & 49.9 & 208.7 & 194.5 & 200.1 & 175.3 & 214.4 & CT & 210.3 \\
\hline 9 & 75 & M & 140 & 7 & MCA (M3) & Left & Yes & No & 29.7 & 55.3 & 52.3 & 48 & 35 & 46.8 & CT & 49.5 \\
\hline 10 & 69 & M & 90 & 8 & MCA (M3) & Left & Yes & No & 25.8 & 101.2 & 74.8 & 84.3 & 84.6 & 90.5 & T2 & 89 \\
\hline 11 & 78 & $\mathrm{M}$ & 60 & 8 & ACA & Right & Yes & No & 29 & 46.2 & 64.1 & 35.3 & 28.6 & 51.3 & $\mathrm{~T} 2$ & 49.7 \\
\hline 12 & 76 & M & 80 & 19 & MCA (M1) & Left & Yes & No & 91.6 & 274.5 & 259.2 & 276 & 222.3 & 269 & T2 & 284.7 \\
\hline 13 & 89 & $\mathrm{~F}$ & 340 & 27 & Basilar & Bilateral & Yes & No & 66.1 & 139.9 & 177.8 & 142.8 & 135.3 & 138.8 & T2 & 131.5 \\
\hline 14 & 79 & $M$ & 240 & 17 & ICA & Left & Yes & No & 35.6 & - & - & - & - & 238.9 & $\mathrm{~T} 2$ & 228.4 \\
\hline 15 & 90 & M & 350 & 13 & ICA & Right & Yes & No & 53.5 & - & - & - & - & 226.1 & CT & 194.2 \\
\hline 16 & 76 & $\mathrm{~F}$ & 300 & 14 & ICA & Right & Yes & No & 16.3 & - & - & - & - & 186 & CT & 217.1 \\
\hline 17 & 53 & M & 240 & 6 & PCA & Right & Yes & No & 37.8 & - & - & - & - & 65.9 & T2 & 59.8 \\
\hline 18 & 68 & M & 290 & 7 & MCA (M3) & Right & Yes & No & 63.5 & - & - & - & - & 104.7 & CT & 74.1 \\
\hline 19 & 37 & $\mathrm{M}$ & 360 & 15 & MCA (M1) & Right & Yes & No & 176.2 & - & - & - & - & 228 & CT & 204.4 \\
\hline 20 & 56 & $\mathrm{M}$ & 310 & 14 & ICA & Left & Yes & No & 84.4 & - & - & - & - & 194.5 & T2 & 171.6 \\
\hline
\end{tabular}

Note:-NIHSS indicates the National Institutes of Health Stroke Scale, ICA, internal carotid artery; MCA, middle cerebral artery; ACA, anterior cerebral artery; PCA, posterior cerebral artery; SVS, susceptibility vessel sign; tPA, tissue plasminogen activator; PWI, perfusion-weighted image; DWI, diffusion-weighted image; TTP, time-to-peak; MTT, mean transit time; rCBF, relative cerebral blood flow; rCBV, relative cerebral blood volume; RMHV on GRE-T2*, region of multiple hypointense vessels on gradient-echo T2*; T2, T2-weighted image; S, sex; GRE, gradient-echo.

$74 \mathrm{~mL}$ (range, $46.8-269 \mathrm{~mL}$ ), and the infarct volume at 72 hours was $214.5 \pm 78.3 \mathrm{~mL}$ (range, $49.5-284.7 \mathrm{~mL}$ ). There was no significant difference between RMHV on GRE-T2* and the infarct volume at 72 hours $(P=.331)$.

\section{Group $3(\mathrm{n}=12)$}

The mean $( \pm \mathrm{SD})$ baseline DWI lesion volume was $85.2 \pm 27.5$ $\mathrm{mL}$ (range, 25.8-106 mL), MTT was 256.3 $\pm 101.1 \mathrm{~mL}$ (range, 52.3-334.8 mL), TTP was $263.3 \pm 99.4 \mathrm{~mL}$ (range, 46.2-329.3 $\mathrm{mL}$ ), CBF was $214.4 \pm 89.3 \mathrm{~mL}$ (range, 35.3-307.9 mL), and CBV was $174.4 \pm 73.7 \mathrm{~mL}$ (range, 28.6-256.1 mL), and RMHV on GRE-T2* was $251.6 \pm 92.6 \mathrm{~mL}$ (range, $46.8-311.5$ $\mathrm{mL})$. There was no significant difference on MTT, TTP, rCBF, and RMHV volume on GRE-T2* $(P=.139)$ (Fig 3 ).

There was a high correlation between examiners performing volume measurements. The ICC values were $0.96(P<$
$.001)$ for DWI, $0.99(P<.001)$ for MTT, $0.99(P<.001)$ for TTP, $0.99(P<.001)$ for rCBF, $0.98(P<.001)$ for rCBV, 0.99 $(P<.001)$ for RMHV on GRE-T2* ${ }^{*}$ and $0.98(P<.001)$ for the infarct volume at 72 hours. RMHV volume on GRE-T2* sequence correlation coefficient values $(r)$ were $0.67(P=.09)$ for DWI, $0.96(P=.001)$ for MTT, $0.92(P=.003)$ for TTP, $0.92(P=.003)$ for $\mathrm{rCBF}, 0.96(P=.001)$ for $\mathrm{rCBV}$, and 0.91 $(P=.001)$ for the infarct volume at 72 hours. NIHSS score correlation coefficient values were $0.55(P=.19)$ for DWI, $0.66(P=.10)$ for MTT, $0.61(P=.14)$ for TTP, $0.61(P=.14)$ for rCBF, $0.68(P=.09)$ for $\mathrm{rCBV}, 0.73(P=.058)$ for $\mathrm{RMHV}$ volume on GRE-T2* ${ }^{\star}$, and $0.73(P=.058)$ for the infarct volume at 72 hours. The Bland-Altman plot shows the agreement between examiners performing ischemic volume measurement on TTP, rCBF, GRE-T2*, and the infarct volume at 72 hours. A total of $100 \%$ of data points were within the bound- 

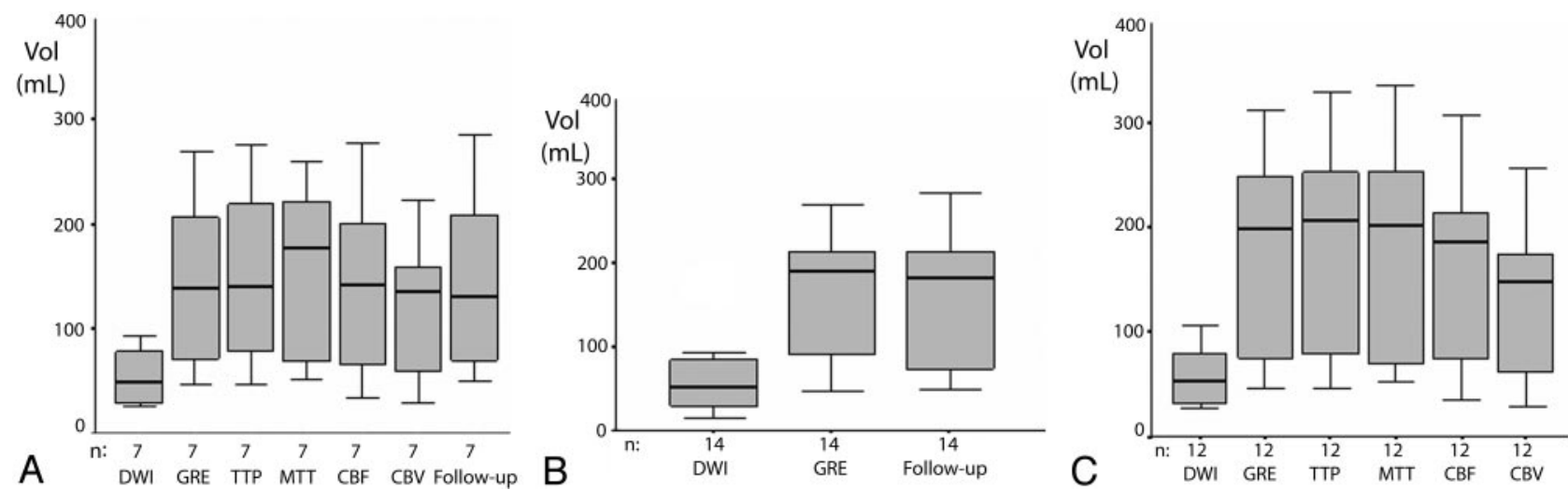

Fig 3. $A$, Baseline DWI, RMHV on GRE-T2* TTP, MTT, rCBF, rCBV, and the infarct volume at 72 hours on follow-up images in group 1. There is no significant difference of volumes among TTP, MTT, rCBF, and RMHV on GRE-T2* and the infarct volume at 72 hours. B, Baseline DWI, RMHV on GRE-T2*, and the infarct volume at 72 hours on follow-up images in group 2. There is no significant difference of volumes between RMHV on GRE-T2* and the infarct volume at 72 hours. C, Volumetric measurement of lesions on baseline DWI, RMHV on GRE-T2*, TTP, MTT, rCBF, and rCBV in group 3. There is no significant difference of volumes among TTP, MTT, rCBF, and RMHV on GRE-T2*.

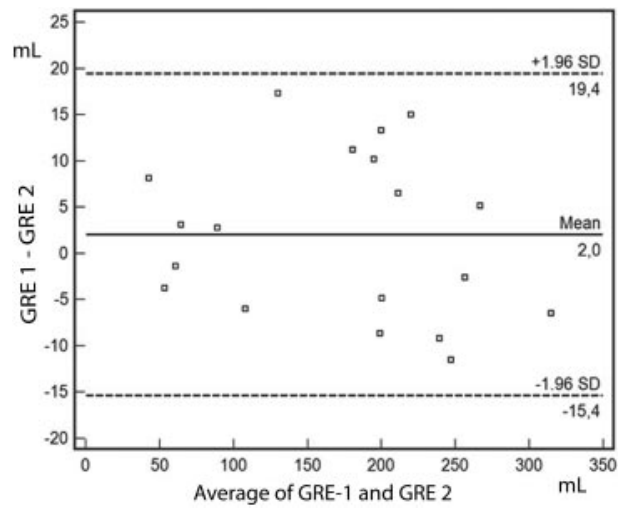

Fig 4. Bland Altman plot of differences between 2 examiners. Dashed lines represent the mean $(1.6 \mathrm{~mL})$ and upper and lower boundaries. A total of $100 \%$ of data points are within the boundary limits for RMHV on GRE-T2*.

ary limits for TTP, MTT, rCBF, RMHV on GRE-T2*, and the infarct volume at 72 hours on follow-up images and $83 \%$ for rCBV (Fig 4). The follow-up MR imaging included GRE-T2* only in 5 patients (patients $10-14$ ) who did not receive thrombolysis. RMHV on GRE-T2* returned to normal within 48 hours after symptom onset in 4 of 5 patients.

\section{Discussion}

The most important finding of our study was that RMHV was identified strictly in the ischemic territory in the hyperacute phase of stroke in all 20 patients on GRE-T2* at 3T. This sign on GRE-T2* can be used as a supportive finding in the radiologic diagnosis of acute stroke. The RMHV volume on GRE$\mathrm{T}_{2}$ s sequence correlated with $\mathrm{MTT}$, TTP, rCBF, and $\mathrm{rCBV}$ lesion volumes on PWI and the infarct volume at 72 hours. There was no significant difference between the ischemic lesion volumes on MTT, TTP, rCBF, RMHV on GRE-T2*, and the infarct volume at 72 hours.

The SVS has been extensively studied in the literature. Cho et $\mathrm{al}^{12}$ have introduced the term SVS to describe the hypointense signal intensity within primarily occluded vessels on GRE-T2* . According to many studies, SVS on GRE-T2* represents a thrombus in an artery analogous to the CT hyperattenuated artery sign. ${ }^{13,14}$ We have also depicted SVS on GRE$\mathrm{T} 2{ }^{*}$ at $3 \mathrm{~T}$ in all 20 patients with acute stroke. Hermier ${ }^{8}$ et al reported "abnormal visualization of leptomeningeal vessels" at $1.5 \mathrm{~T}$ GRE in 17 of 48 patients with hemispheric ischemic stroke, which had no impact on clinical status and on the late infarct volume. We observed a similar finding of multiple hypointense vessels confined to the ischemic territory and that was not visible on the normal contralateral hemisphere, for which we preferred to use the term $R M H V$ to emphasize this area containing multiple small venous or arterial vessels.

Increased oxygen extraction fraction in an ischemic brain with reduced cerebral blood flow ${ }^{15,16}$ leads to increased blood deoxyhemoglobin concentration in the cerebral veins and capillaries. Venous plus capillary fraction of cerebral blood volume is $63 \%$ to $70 \%$ of the total cerebral blood volume and contains higher concentrations of deoxyhemoglobin. ${ }^{17}$ Slow flow state may also contribute to an increased level of deoxyhemoglobin in the ischemic territory. ${ }^{18}$ The magnetic susceptibility difference between venous blood and the surrounding brain tissue makes it possible to visualize venous structures with use of GRE-T2* sequences. ${ }^{19}$ Reichenbach et $\mathrm{al}^{20}$ have demonstrated that multiple hypointense structures on MR venography at $3 \mathrm{~T}$ were considered to be of mostly venous origin according to their anatomic distribution. However, multiple hypointense vascular structures may also include dilated capillaries or arterioles in the ischemic territory. Whether these vessels are dilated should be decided by angiography.

Prolongation of MTT secondary to slow flow has been observed as an early sign of ischemia such as in our series. ${ }^{21}$ RMHV volume on GRE-T2* was found to be larger than the DWI lesion volume within 6 hours and mostly disappeared within 48 hours (subacute phase) after symptom onset. There was no significant correlation between RMHV and NIHSS. Although there was no statistically significant difference in calculated lesion volumes on MTT, TTP, rCBF, RMHV on GRE-T2* ${ }^{*}$ and the infarct volume at 72 hours, the results of our volumetric measurements showed minor variation. Baseline RMHV volume on GRE-T2* was smaller than baseline MTT and TTP lesion volumes. It has been already shown that $\mathrm{MTT}^{22}$ and $\mathrm{TTP}^{23}$ maps tend to overestimate the volume of tissue at risk for infarction.

PWI is a quantitative imaging technique for the prediction of final infarct volume. ${ }^{24}$ Our results showed that GRE-T2* can be used as an adjunctive or additive sequence to PWI. For 
example, patient 5 was admitted to our hospital within 80 minutes, presenting with a right-sided mild hemiparesis (NIHSS: 6). She had an acute occlusion of the left angular branch (M3) of the MCA with concomitant ipsilateral severe ICA stenosis. The DWI lesion volume of this patient was 6.1 $\mathrm{mL}$, TTP was $281.4 \mathrm{~mL}, \mathrm{rCBF}$ was $176.9 \mathrm{~mL}$, and RMHV on GRE-T2* was $70.7 \mathrm{~mL}$. We realized that acute occlusion of the M3 branch in this patient could not be responsible for the large hemispheric hypoperfusion. In conditions that are not straightforward, the GRE-T2* sequence may be helpful to determine the exact nature of an unperfused ischemic area.

Recent evidence suggests a potential link between the administration of gadolinium-containing contrast agents and nephrogenic systemic fibrosis/nephrogenic fibrosing dermopathy in patients with renal failure. ${ }^{25}$ GRE-T2* sequence can be a better choice in patients who have renal disease or a history of hypersensitivity to contrast agents. From a clinical point of view, RMHV on GRE-T2* depicts a region of hypoperfused tissue in acute stroke without the use of MR imaging contrast agents. Our data show that within a clinical routine setting of acute ischemic stroke evaluation, additional GRE-T2* sequence can supply an increased safety profile for the DWI/ PWI mismatch concept. Combined DWI, PWI, and GRE-T2* sequences may produce valuable information in understanding the pathophysiology and treatment strategies of acute ischemic stroke. Haacke et $\mathrm{al}^{26}$ presented a new method of MR imaging, which they refer to as susceptibility-weighted imaging (SWI). The SWI technique is a high spatial resolution accelerated 3D T2*-weighted GRE sequence and has an advantage of depicting and enhancing magnetic susceptibility effects better than conventional T2*-weighted GRE sequence. ${ }^{27}$ This technique was unavailable during the period we performed our study.

Our study had some limitations. Although 1 month is the ideal time for final follow-up of infarct, ${ }^{28}$ we used 72 hours of follow-up imaging because most of our patients had acute large cerebral artery occlusions with severe disability. We preferred to perform imaging studies on those patients before they left the hospital. The infarct volume at 72 hours for patients who received thrombolysis was not included in the statistical analysis because the rtPA induced an improved effect on ischemic penumbra in the hyperacute phase. Seven patients outside of the thrombolysis window did not receive perfusion imaging. Furthermore, the number of our cases of stroke may be somewhat small, but of 67 cases, 20 patients who met the inclusion criteria and who were examined with advanced techniques within 6 hours were admitted to the study.

\section{Conclusions}

Our study indicates that the RMHV on GRE-T2* at $3 \mathrm{~T}$ can be used as a supportive diagnostic imaging finding in acute ischemic stroke and that GRE-T2* is a useful sequence that provides information in accordance with PWI data.

\section{References}

\footnotetext{
1. Ogawa S, Lee TM, Kay AR, et al. Brain magnetic resonance imaging with contrast dependent on blood oxygenation. Proc Natl Acad Sci U S A 1990;87:9868-72
}

2. Reichenbach JR, Venkatesan R, Schillinger DJ, et al. Small vessels in the human brain: MR venography with deoxyhemoglobin as an intrinsic contrast agent. Radiology 1997;204:272-77

3. Kidwell CS, Chalela JA, Saver JL, et al. Comparison of MRI and CT for detection of acute intracerebral hemorrhage. JAMA 2004;292:1823-30

4. Schellinger PD, Chalela JA, Kang DW, et al. Diagnostic and prognostic value of early MR imaging vessel sign in hyperacute stroke patients imaged $<3$ hours and treated with recombinant tissue plasminogen activator. AJNR Am J Neuroradiol 2005;26:618-24

5. Roussel SA, van Bruggen N, King MD, et al. Identification of collaterally perfused areas following focal cerebral ischemia in the rat by comparison of gradient echo and diffusion-weighted MRI. J Cereb Blood Flow Metab $1995 ; 15: 578-86$

6. Tamura H, Hatazawa J, Toyoshima H, et al. Detection of deoxygenation-related signal change in acute ischemic stroke patients by $\mathrm{T} 2{ }^{\star}$-weighted magnetic resonance imaging. Stroke 2002;33:967-71

7. Hermier M, Nighoghossian N, Derex L, et al. Hypointense transcerebral veins at $\mathrm{T} 2{ }^{*}$-weighted MRI: a marker of hemorrhagic transformation risk in patients treated with intravenous tissue plasminogen activator. J Cereb Blood Flow Metab 2003;23:1362-70

8. Hermier M, Nighoghossian N, Derex L, et al. Hypointense leptomeningeal vessels at $\mathrm{T} 2{ }^{*}$-weighted MRI in acute ischemic stroke. Neurology 2005;65:652-53

9. Tissue plasminogen activator for acute ischemic stroke. The National Institute of Neurological Disorders and Stroke rt-PA Stroke Study Group. N Engl J Med 1995;333:1581-87

10. Bland JM, Altman DG. Statistical methods for assessing agreement between two methods of clinical measurment. Lancet 1986;8476:307-10

11. Ay H, Arsava M, Vangel M, et al. Interexaminer difference in infarct volume measurements on MRI. A source of variance in stroke research. Stroke 2008;39:1171-76

12. Cho KH, Kim JS, Kwon SU, et al. Significance of susceptibility vessel sign on $\mathrm{T} 2{ }^{*}$-weighted gradient echo imaging for identification of stroke subtypes. Stroke 2005;36:2379-83

13. Kim HS, Lee DH, Choi CG, et al. Progression of middle cerebral artery susceptibility sign on $\mathrm{T} 2{ }^{\star}$-weighted images: its effect on recanalization and clinical outcome after thrombolysis. AJR Am J Roentgenol 2006;187:W650-57

14. Flacke S, Urbach H, Keller E, et al. Middle cerebral artery (MCA) susceptibility sign at susceptibility-based perfusion MR imaging: clinical importance and comparison with hyperdense MCA sign at CT. Radiology 2000;215:476-82

15. Baron JC, Bousser MG, Rey A, et al. Reversal of focal "misery-perfusion syndrome" by extra-intracranial arterial bypass in hemodynamic cerebral ischemia. A case study with 150 positron emission tomography. Stroke 1981;12:454-59

16. Lee JM, Vo KD, An H, et al. Magnetic resonance cerebral metabolic rate of oxygen utilization in hyperacute stroke patients. Ann Neurol 2003;53:227-32

17. Ito $\mathrm{H}$, Kanno I, Iida $\mathrm{H}$, et al. Arterial fraction of cerebral blood volume in humans measured by positron emission tomography. Ann Nucl Med 2001;15:111-16

18. Kennan RP, Zhong J, Gore JC. Intravascular susceptibility contrast mechanisms in tissues. Magn Reson Med 1994;31:9-21

19. Reichenbach JR, Haacke EM. High-resolution BOLD venographic imaging: a window into brain function. NMR Biomed 2001;14:453-67

20. Reichenbach JR, Barth M, Haacke EM, et al. High-resolution MR venography at 3.0 Tesla. J Comput Asist Tomogr 2000;24:949-57

21. Takasawa M, Jones PS, Guadagno JV, et al. How reliable is perfusion MR in acute stroke? Validation and determination of the penumbra threshold against quantitative PET. Stroke 2008;39:870-77

22. Rivers CS, Wardlaw JM, Armitage PA, et al. Do acute diffusion- and perfusionweighted MRI lesions identify final infarct volume in ischemic stroke? Stroke 2006;37:98-104

23. Shih LC, Saver JL, Alger JR, et al. Perfusion-weighted magnetic resonance imaging thresholds identifying core, irreversibly infarcted tissue. Stroke 2003;34:1425-30

24. Sorensen AG, Copen WA, Ostergaard L, et al. Hyperacute stroke: simultaneous measurement of relative cerebral blood volume, relative cerebral blood flow, and mean tissue transit time. Radiology 1999;210:519-27

25. Broome DR, Girguis MS, Baron PW, et al. Gadodiamide-associated nephrogenic systemic fibrosis: why radiologists should be concerned. AJR Am J Roentgenol 2007;188:586-92

26. Haacke EM, Xu Y, Cheng YC, et al. Susceptibility weighted imaging (SWI). Magn Reson Med 2004;52:612-18

27. Tong KA, Ashwal S, Obenaus A, et al. Susceptibility-weighted MR imaging: A review of clinical applications in children. AJNR Am J Neuroradiol 2008;29:9-17

28. Beaulieau C, de Crespigny A, Tong DC, et al. Longitudinal magnetic resonance imaging study of perfusion and diffusion in stroke: evolution of lesion volume and correlation with clinical outcome. Ann Neurol 1999;46:568-78 